\title{
Defective insulin signaling and the protective effects of dimethyldiguanide during follicular development in the ovaries of polycystic ovary syndrome
}

\author{
FAN WANG $^{1 *}$, SHAOBING WANG ${ }^{2,3^{*}}$, ZHENGHONG ZHANG $^{1-3}$, QINGQIANG LIN ${ }^{1}$, \\ YIPING LIU ${ }^{2,3}$, YIJUN XIAO ${ }^{1}$, KAIZHUAN XIAO $^{1}$ and ZHENGCHAO WANG ${ }^{1-3 *}$ \\ ${ }^{1}$ Provincial Key Laboratory for Developmental Biology and Neurosciences, College of Life Sciences; \\ ${ }^{2}$ State Key Laboratory for Evaluation of Exercise Physiological Functions from General Administration of Sport of China, \\ School of Physical Education and Sport Sciences; ${ }^{3}$ Laboratory of Sport Physiology and Biomedicine, School of \\ Physical Education and Sport Sciences, Fujian Normal University, Fuzhou, Fujian 350007, P.R. China
}

Received January 14, 2017; Accepted August 10, 2017

DOI: $10.3892 / \mathrm{mmr} .2017 .7678$

\begin{abstract}
It is established that the physiological effects of insulin are primarily mediated by the insulin signaling pathway. However, a defective insulin signaling is closely associated with the clinical manifestations of polycystic ovary syndrome (PCOS), which include excess androgen levels, insulin resistance and anovulation, and is involved in the pathophysiology of PCOS at the molecular level. Dimethyldiguanide (DMBG) has been widely employed to alleviate reproduction dysfunction in women with PCOS, however, the exact mechanism of this effect remains unclear. The objective of the present study was to investigate the effects of DMBG on the expression of the insulin signaling pathway in the ovaries of rats with PCOS, and to identify the potential underlying molecular mechanisms of these effects in PCOS. In the present study, a PCOS rat model was induced by letrozole, and successful establishment of the model was confirmed by examining ovarian histology and determining serum testosterone levels, by hematoxylin and eosin staining and ELISA, respectively. Subsequently, the expression of two key elements of insulin signaling, insulin receptor substrate (IRS)-2 and phosphatidylinositol 3-kinase (PI3K), was determined by immunohistochemistry and western blot analysis. The results demonstrated that IRS-2 and PI3K expression was markedly decreased in PCOS ovaries, which was rescued by
\end{abstract}

Correspondence to: Dr Zhengchao Wang, Provincial Key Laboratory for Developmental Biology and Neurosciences, College of Life Sciences, Fujian Normal University, 8 Shangsan Road, Fuzhou, Fujian 350007, P.R. China

E-mail: zcwang@fjnu.edu.cn

${ }^{*}$ Contributed equally

Key words: dimethyldiguanide, insulin signaling pathway, polycystic ovary syndrome, ovary, rat
DMBG treatment. These results indicate that IRS-2/PI3K signaling may be involved in the development of PCOS and the therapeutic effects of DMBG on PCOS. To further confirm the effects of DMBG on insulin signaling expression during this process, the expression of an additional two downstream proteins, phosphoinositide-dependent kinase-1 (PDK-1) and the mammalian target of rapamycin (mTOR), was also investigated in the present study, and the results demonstrated that the expression of PDK-1 and mTOR was significantly reduced in PCOS ovaries and increased following DMBG treatment, further indicating that altered insulin signaling may have an important role in the development and treatment of PCOS. In conclusion, the results of the present study indicate that the reduced expression of proteins involved in insulin signaling may contribute to the development of the clinical features of PCOS, and DMBG reverses reduced expression of insulin signaling components, by a mechanism that is yet to be determined, to attenuate certain symptoms of PCOS, such as obesity. To the best of our knowledge, the present study is the first to provide data regarding the detailed changes of insulin signaling during the development and treatment of PCOS, and may provide an important reference for clinical PCOS treatment.

\section{Introduction}

Polycystic ovary syndrome (PCOS) is one of the most common reproductive endocrine diseases among women of childbearing age; PCOS is affected by various factors and there are no unique diagnostic criteria in different regions, due to the heterogeneity of clinical manifestations and endocrine system changes of PCOS $(1,2)$. Therefore, the precise pathophysiology of PCOS remains to be determined. An increasing number of studies have demonstrated that the insulin signaling pathway has an important role in the pathophysiology of PCOS, including phosphatidylinositol 3-kinase (PI3K) and protein kinase B/Akt signaling, which is critically implicated in insulin resistance, androgen secretion, obesity and follicular development (3-10). 
PCOS manifests as defective ovarian steroid biosynthesis and hyperandrogenemia, and $50-70 \%$ of women with PCOS exhibit insulin resistance and are hyperinsulinemic (9-11), indicating that insulin resistance and hyperinsulinism may have an important role in the pathophysiology of PCOS. Hyperinsulinemia is reported to be associated with excessive androgens levels and anovulation (9-11). Insulin exerts effects on the ovary via its own receptor and interacts with gonadotrophins to regulate steroidogenesis. Furthermore, numerous studies have demonstrated that selective insulin resistance may develop, where the metabolic response to insulin action is impaired, such as the PI3K pathway, while other functions are not only retained but may be enhanced, such as the mitogen-activated protein kinase (MAPK) pathway, in classic insulin target tissues and in the ovary (3-5). Selective ovary resistance is considered to be a selective defect in insulin action in the PCOS ovary, which affects the metabolic, and not the mitogenic, actions of insulin (12-14).

Insulin resistance and excessive androgen levels are associated with a defective PI3K pathway and not the MAPK cascade (3-5). Inhibition of PI3K in the ovary leads to reduced activation of its downstream signaling, which affects the positive actions of insulin-induced glucose uptake (15). It is established that the physiological role of insulin occurs primarily via the insulin signaling pathway. Insulin binds to its receptor and activates insulin receptor substrate (IRS) via the phosphorylation of tyrosine residues. PI3K subsequently interacts with the phosphorylated tyrosine residues on IRS molecules, which leads to the phosphorylation of phosphatidyl inositol-4,5-diphosphate (PIP2) to produce phosphatidyl inositol-3,4,5-triphosphate (PIP3) as a secondary messenger, and subsequently activates phosphoinositide-dependent kinase 1 (PDK-1) (6-10). Upon activation of the PI3K pathway, signaling may be propagated to a diverse array of substrates, such as mammalian target of rapamycin (mTOR) (3-5,14-16).

Dimethyldiguanide (DMBG), a common insulin-sensitizing agent for the control of glycemia in type 2 diabetes, is also reported to alleviate the clinical symptoms of women with PCOS (17-23). DMBG administration is beneficial for lowering hyperinsulinemia and hyperandrogenemia, and restoring ovulation in certain cases by enhancing glucose uptake, thus leading to reduced synthesis/secretion of insulin (9-11). An increasing number of studies have demonstrated that DMBG modulates the insulin sensitivity and glucose metabolism of its target tissues in women with PCOS $(24,25)$. DMBG is also reported to increase the levels of molecules that regulate the expression of the insulin-dependent glucose transporter GLUT4 in the endometria of hyperinsulinemic patients with PCOS (25). Therefore, the present study determined the expression levels of insulin signaling molecules in the ovaries of rats with PCOS with and without DMBG treatment, to demonstrate defective insulin signaling in the ovaries during PCOS and the protective effects of DMBG. The present study may provide novel insight for further investigation of the regulatory mechanism during the development and treatment of PCOS.

\section{Materials and methods}

Animals. Sprague-Dawley rats were purchased from Wushi Experimental Animal Supply Co., Ltd. (Fuzhou, China). The animals were maintained at $23-26^{\circ} \mathrm{C}$ and $50-70 \%$ relative humidity under a $14 \mathrm{~h}$ light/10 h dark schedule with a continuous supply of chow and water. The experimental protocol was approved by the Institutional Animal Care and Use Committee and Research Ethics Committee of Fujian Normal University (Fuzhou, China).

Experimental design. Female 6-week-old rats ( $\mathrm{n}=40$; mean body weight, $157 \pm 10 \mathrm{~g}$ ) with two consecutive 4-day estrous cycles were randomly divided into four groups, including blank control, vehicle control, PCOS model and DMBG treatment groups. The rats in PCOS model and DMBG groups were induced by intragastric administration of $1 \mathrm{mg} / \mathrm{kg} /$ day letrozole dissolved in $1 \%$ carboxymethyl cellulose (CMC; $2 \mathrm{ml} / \mathrm{kg}$ ) for 21 days, while the vehicle control was administered with an equal amount of CMC. Following the treatment with letrozole for 21 days, rats in the DMBG group received 300 mg/kg DMBG (Sangon Biotech Co., Ltd., Shanghai, China) by intragastric infusion for 4 weeks. The body weight was recorded throughout the experimental period. Following treatments, all rats were sacrificed and the ovaries were removed. The left ovary of the experimental animals was fixed in $4 \%$ paraformaldehyde for 2 days at room temperature and used for ovarian histological examination and immunohistochemistry; the right ovary was frozen at $-80^{\circ} \mathrm{C}$ and used for detecting the expression of functional proteins. Blood samples ( $3 \mathrm{ml})$ were collected from the heart and allowed to stand for $30 \mathrm{~min}$ at room temperature, and then centrifuged at $1,000 \mathrm{xg}$ for $10 \mathrm{~min}$ at $4^{\circ} \mathrm{C}$. The prepared serum was stored at $-80^{\circ} \mathrm{C}$ for examination of testosterone levels. The experiment was repeated two times.

Determination of serum testosterone levels. The concentration of serum testosterone was examined by an ELISA kit (cat. no. hj-462; Xiamen Huijia Biotechnology Co., Xiamen, China), according to the manufacturer's protocol.

Hematoxylin and eosin staining for ovarian histology. The left ovary of rats was fixed in $4 \%$ paraformaldehyde and embedded in paraffin. Subsequently, $5 \mu \mathrm{m}$ sections were cut and mounted on slides. The sections were stained using a hematoxylin and eosin staining kit (Beyotime Institute of Biotechnology, Haimen, China) and performed according to the manufacturer's protocol. The images were obtained using a light microscope (BX51; Olympus Corporation, Tokyo, Japan).

Immunohistochemistry of IRS-2 and PI3K. Paraformaldehydefixed paraffin-embedded left ovary sections $(5 \mu \mathrm{m})$ were also processed by immunohistochemical analysis with IRS-2 (cat. no. bs-0173R; 1:200) and PI3K (cat. no. bs-0128R; 1:200; both from BIOSS, Beijing, China) primary antibodies. The sections were deparaffinized at room temperature in $100 \%$ dimethylbenzene for $2 \times 10 \mathrm{~min}$ and rehydrated in descending alcohol series $(100,90,80,70,50,30$ and $0 \%)$ for 5 min each. Antigen retrieval was performed using sodium citrate buffer ( $\mathrm{pH}$ 6.0) by microwave heating at $95^{\circ} \mathrm{C}$ for $5 \mathrm{~min}$. The sections were allowed to cool to room temperature, washed for $3 \times 2 \mathrm{~min}$ in PBS and incubated at room temperature for 15 min with $3 \%$ hydrogen peroxide to block the endogenous peroxidase. Protein block was performed with $10 \%$ bovine serum albumin (BSA; 
Beyotime Institute of Biotechnology) at room temperature for $30 \mathrm{~min}$. Sections were incubated at $4^{\circ} \mathrm{C}$ overnight with primary antibodies, and the primary antibody immunoreactivity was visualized using an Elite ABC kit (cat. no. PK-6101; BioGenex, San Ramon, CA, USA) according to the manufacturer's instructions. Briefly, the sections were washed for $3 \times 2 \mathrm{~min}$ in PBS, incubated for $25 \mathrm{~min}$ at room temperature with diluted biotinylated secondary antibody, then incubated for $30 \mathrm{~min}$ at room temperature with a mixture of reagent $\mathrm{A}$ and reagent $\mathrm{B}$. The negative control was examined using $1 \%$ BSA instead of primary antibody. Sections were subsequently counterstained with hematoxylin at room temperature for $4 \mathrm{~min}$ and mounted with coverslips to determine the structure and types of cells in the rat ovary under the BX51 light microscope.

Western blot analysis of IRS-2, PI3K, PDK-1 and mTOR protein expression. The right ovaries of the rats were lysed on ice using radioimmunoprecipitation assay lysis buffer (cat. no. P0013B) with phenylmethanesulfonyl fluoride (cat. no. ST506; both from Beyotime Institute of Biotechnology) for $15 \mathrm{~min}$, and the lysate was centrifuged at $13,000 \mathrm{x} \mathrm{g}$ at $4^{\circ} \mathrm{C}$ for $10 \mathrm{~min}$. Protein concentration was determined by a BCA assay kit (Beyotime Institute of Biotechnology). Protein samples $(20 \mu \mathrm{g})$ were subjected to $8 \%$ SDS-PAGE and electrophoretically transferred onto a polyvinylidene fluoride membrane. The membrane was blocked at room temperature with 5\% nonfat milk for $45 \mathrm{~min}$, and then washed and probed with IRS-2 antibody (cat. no. bs-0173R, 1:500; BIOSS) overnight at $4^{\circ} \mathrm{C}$. After washing, the membrane was incubated with horseradish peroxidase-labeled goat anti-rabbit $\operatorname{IgG}$ (cat. no. A0208; 1:2,000; Beyotime Institute of Biotechnology) for $60 \mathrm{~min}$ at room temperature, and the membrane was developed using a BeyoECL Plus kit (P0018; Beyotime Institute of Biotechnology). The membrane was subsequently incubated with stripping buffer (cat. no. P0025; Beyotime Institute of Biotechnology) at room temperature for $2 \mathrm{~min}$, washed with distilled water for $5 \mathrm{~min}$, blocked as aforementioned and reprobed overnight at $4^{\circ} \mathrm{C}$ using the following antibodies: Anti-PI3K p85a (cat. no. bs-0128R; 1:500; BIOSS); anti-PDK-1 (cat. no. 18262-1-AP; 1:1,000; Wuhan Sanying Biotechnology, Wuhan, China); anti-mTOR (cat. no. 20657-I-AP; 1:1,000; Wuhan Sanying Biotechnology); anti- $\beta$-actin (cat. no. sc-130656; 1:5,000; Santa Cruz Biotechnology, Inc., Dallas, TX, USA). The densitometry of each band was analyzed using Image Pro Plus 6.0 (Media Cybernetics, Inc., Rockville, MD, USA).

Statistical analysis. Experiments were repeated six times and data were presented as the mean + standard error of the mean. The significance of differences between the mean values among and between multiple groups was evaluated using one-way analysis of variance followed by a Tukey's multiple comparisons test. Analyses were performed using SPSS version 19.0 (SPSS, Inc., Chicago, IL, USA). P $<0.05$ was considered to indicate a statistically significant difference.

\section{Results}

Alterations in body weight and serum testosterone levels. PCOS was identified by examining the differences in body weight and serum testosterone levels. The results demonstrated
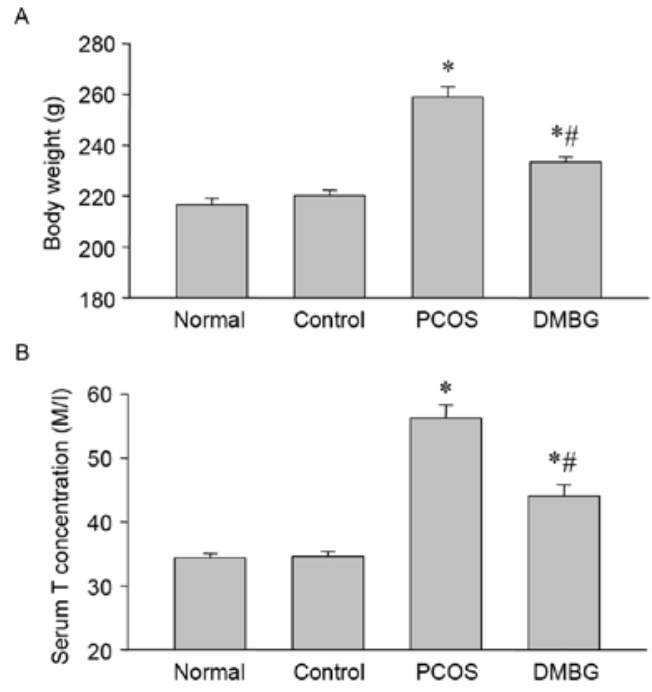

Figure 1. Differences in body weight and serum testosterone levels (A) Differences in body weight among groups were examined at the end of experiments (B) Serum testosterone concentrations were detected by ELISA in each group. Data are presented as the mean \pm standard error of the mean. ${ }^{*} \mathrm{P}<0.05$ vs. blank control group; ${ }^{\#} \mathrm{P}<0.05$ vs. PCOS group. PCOS, polycystic ovary syndrome; DMBG, dimethyldiguanide; $\mathrm{T}$, testosterone.

that the body weight of PCOS rats was markedly higher compared with the blank and vehicle control groups (Fig. 1A), while DMBG treatment reduced this increase of body weight in PCOS rats (Fig. 1A). The results also indicated a significant increase in the serum testosterone levels of PCOS rats compared with blank control rats (Fig. 1B), which was consistent with previous reports $(19,22,23)$. Notably, DMBG also decreased the levels of serum testosterone in rats with PCOS (Fig. 1B). These results indicate that the PCOS model was developed successfully and that DMBG may alleviate certain symptoms of PCOS, including weight gain and excessive androgen levels.

Protective effects of DMBG on the follicular development in PCOS ovaries as determined by histological examination. To further confirm PCOS model establishment and the effects of DMBG, the present study examined histological changes in ovaries by hematoxylin and eosin staining. The results demonstrated that healthy structures of follicles in different development stages and the corpus luteum were observed in the blank control (Fig. 2A) and vehicle control (Fig. 2B) groups. Compared with the two control groups, the majority of follicles in PCOS rats were expanding cystic follicles with degrading granulosa cell layers (Fig. 2C), while the number of expanding cystic follicles was decreased in DMBG-treated rats compared with the PCOS group, and the presence of a number of healthy follicles and corpus luteum was observed (Fig. 2D). Higher magnification images for blank control, vehicle control, PCOS and DMBG groups are provided in Fig. 2E-H.

Effects of DMBG on the expression of IRS-2 and PI3K protein in the ovaries by immunohistochemical examination. It is established that IRS-2 has an important role in insulin signaling transduction during the initial stage. The present study therefore determined the protein expression of IRS-2 in ovaries from each group by immunohistochemistry (Fig. 3). 

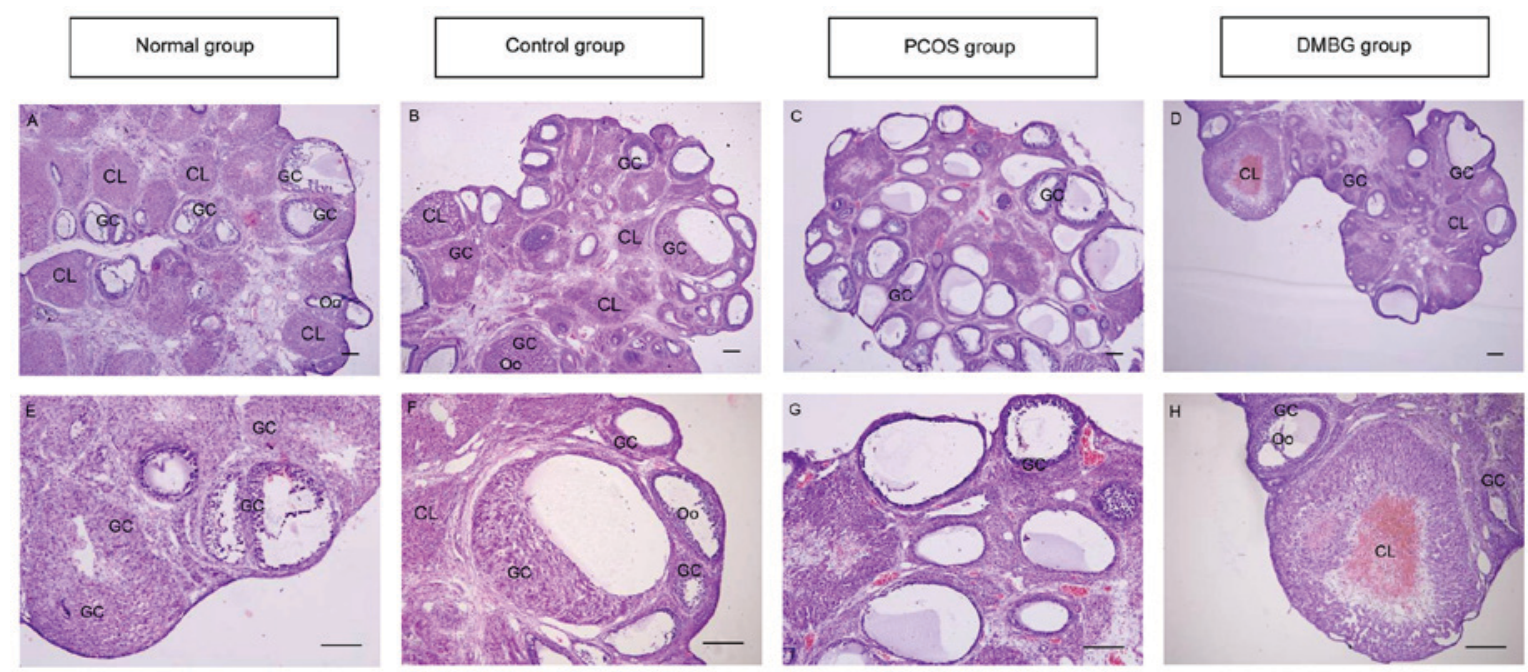

Figure 2. Ovarian histological examination. Histological sections were stained with hematoxylin and eosin. Follicles developed normally in the (A) blank control and (B) vehicle control groups (C) Numerous follicular cysts were present in PCOS ovaries with degrading granulosa cells in the thin layer of granulosa cells. (D) DMBG treatment rescued follicular development. Higher magnification images are presented for (E) blank control, (F) vehicle control, (G) PCOS and (H) DMBG groups. Scale bar, $100 \mu \mathrm{m}$. PCOS, polycystic ovary syndrome; DMBG, dimethyldiguanide; GC, granulosa cell, Oo, oocyte, CL, corpus luteum.
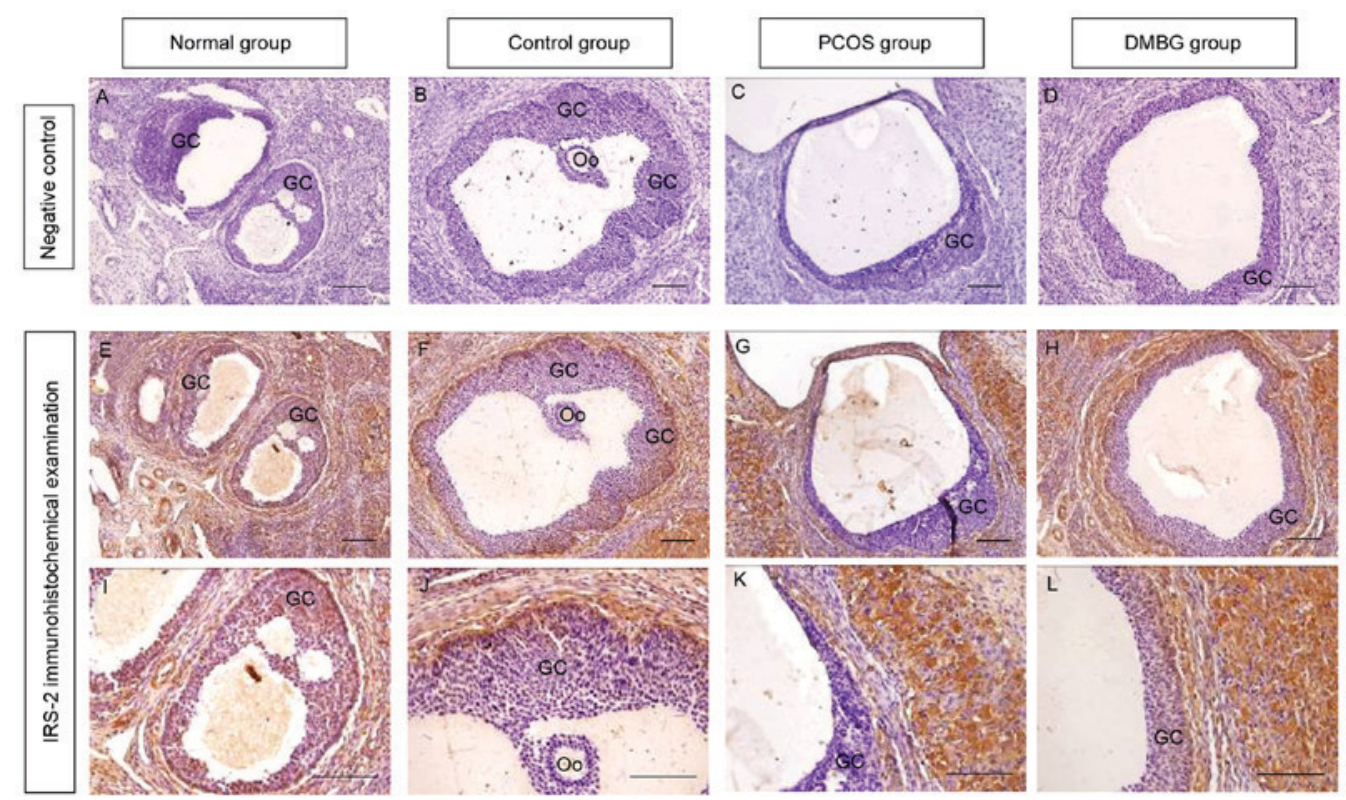

Figure 3. Immunohistochemical examination of IRS-2 expression in the ovaries of each group. Negative control staining with serum instead of IRS-2 primary antibody in (A) blank control, (B) vehicle control, (C) PCOS and (D) DMBG groups. IRS-2 immunohistochemical signals appear brown and the counterstaining background appears blue in (E) blank control, (F) vehicle control, (G) PCOS and (H) DMBG groups. Higher magnification images of IRS-2 staining are presented for (I) blank control, (J) vehicle control, (K) PCOS and (L) DMBG groups. Scale bar, $100 \mu \mathrm{m}$. IRS, insulin receptor substrate; PCOS, polycystic ovary syndrome; DMBG, dimethyldiguanide; GC, granulosa cell; Oo, oocyte.

The results demonstrated that IRS- 2 was expressed in all types of ovarian cells and its expression level was decreased in the granulosa cells of PCOS rats compared with the two controls. Notably, DMBG rescued IRS-2 expression and follicular development in the ovaries of PCOS rats (Fig. 3). Upon activation, IRS-2 binds to the $\mathrm{SH} 2$ domain of the $\mathrm{p} 85$ regulatory subunit of PI3K (8). To further understand the effects of insulin signaling, the present study also determined the expression of the PI3K regulator subunit in the ovaries of each group by immunohistochemistry (Fig. 4). The results demonstrated that PI3K expression was also decreased in PCOS rats, similar to the results for IRS-2 expression. In addition, DMBG rescued this reduction of PI3K expression in DMBG-treated PCOS rats (Fig. 4). These results indicate that IRS-2/PI3K signaling may be involved in the development and treatment of PCOS.

$D M B G$ reverses alterations in the protein expression of IRS-2 and PI3K in the ovaries, as determined by western blotting. To further confirm the immunohistochemical results, the protein expression of IRS-2 and PI3K were also examined by western blot analysis. The results demonstrated a marked decrease in IRS-2 and PI3K expression in PCOS rats compared with the blank and vehicle control groups (Fig. 5). Furthermore, DMBG partially reversed these decreases in IRS-2 and PI3K 

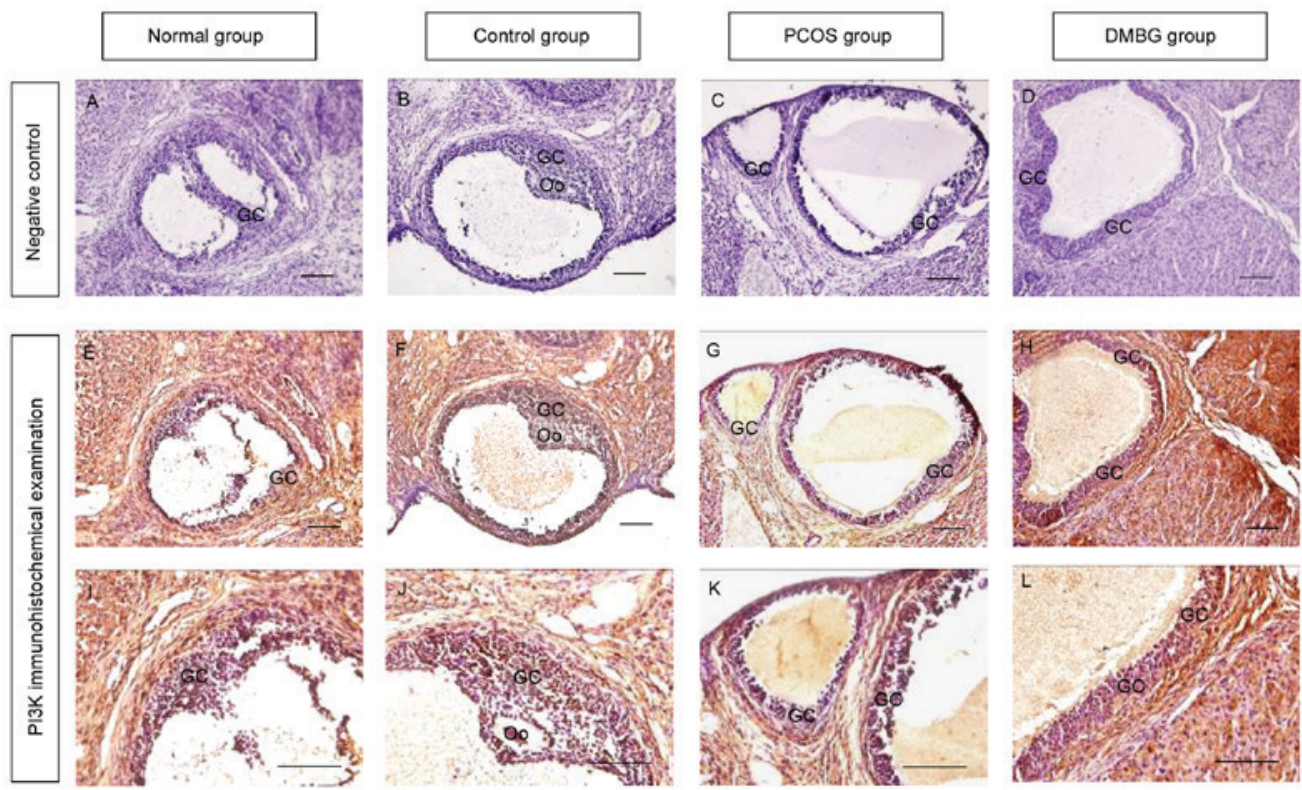

Figure 4. Immunohistochemical examination of PI3K expression in the ovaries of each group. Negative control staining with serum instead of PI3K primary antibody in (A) blank control, (B) vehicle control, (C) PCOS and (D) DMBG groups. PI3K immunohistochemical signals appear brown and the counterstaining background appears blue in (E) blank control, (F) vehicle control (G) PCOS and (H) DMBG groups. Higher magnification images of PI3K staining are presented for (I) blank control, (J) vehicle control, (K) PCOS and (L) DMBG groups. Scale bar, $100 \mu \mathrm{m}$. PI3K, phosphatidylinositol 3-kinase; PCOS, polycystic ovary syndrome; DMBG, dimethyldiguanide; GC, granulosa cell; Oo, oocyte.

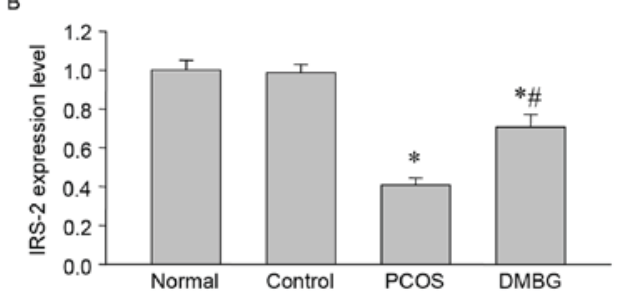

C

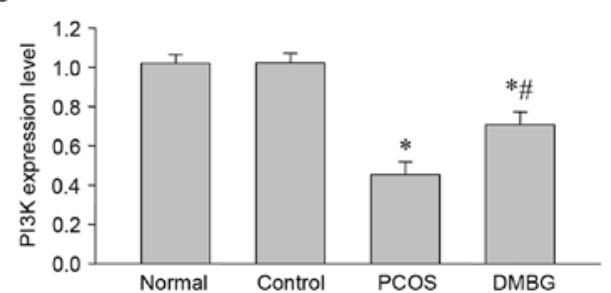

Figure 5. Western blot analysis of IRS-2 and PI3K protein expression in the ovaries of each group. (A) Representative western blot demonstrating the protein expression of IRS-2 and PI3K. Quantified levels of (B) IRS-2 and (C) PI3K protein normalized to the blank control. Data are presented as the mean \pm standard error of the mean, $\mathrm{n}=6 .{ }^{*} \mathrm{P}<0.05$ vs. blank control group; ${ }^{\sharp} \mathrm{P}<0.05$ vs. PCOS group. IRS, insulin receptor substrate; PI3K, phosphatidylinositol 3-kinase; PCOS, polycystic ovary syndrome; DMBG, dimethyldiguanide.

expression (Fig. 5), indicating defective IRS-2/PI3K signaling and potential protective effects of DMBG in PCOS.

PDK-1 and mTOR protein expression is reduced in PCOS ovaries and reversed by DMBG treatment, as determined

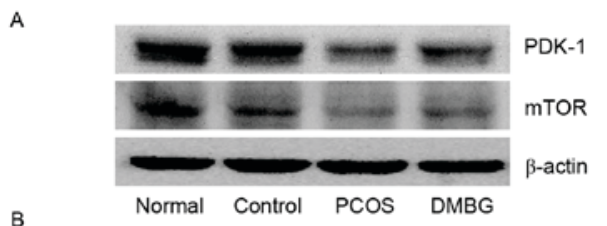

B
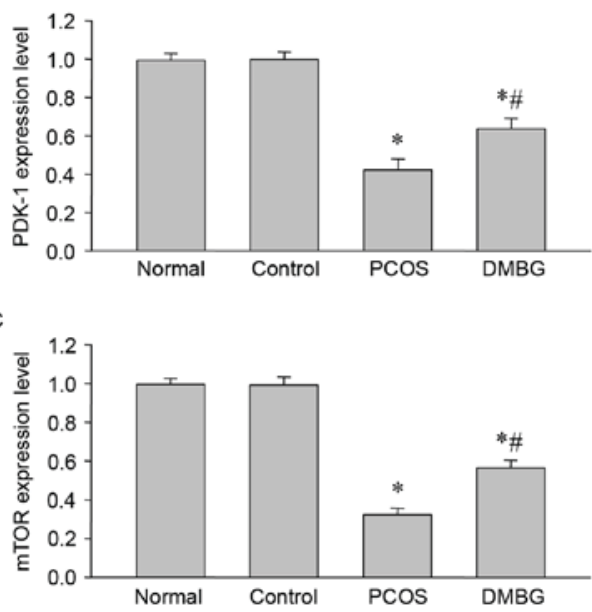

Figure 6. Western blot analysis of PDK-1 and mTOR protein expressions in the ovaries of each group. (A) Representative western blot demonstrating the protein expression of PDK-1 and mTOR. Quantified levels of (B) PDK-1 and $(C)$ mTOR protein normalized to the blank control. Data are presented as the mean \pm standard error of the mean, $n=6$. ${ }^{*} \mathrm{P}<0.05$ vs. blank control group; ${ }^{\text {P }}<0.05$ vs. PCOS group. PDK-1, phosphoinositide-dependent kinase-1; mTOR, mammalian target of rapamycin; PCOS, polycystic ovary syndrome; DMBG, dimethyldiguanide.

by western blotting. As PDK-1 and mTOR are downstream proteins of IRS-2/PI3K signaling, the present study also performed western blot analysis to determine the protein expression of PDK-1 and mTOR in the ovaries from each group (Fig. 6). Notably, the expression of PDK-1 and mTOR 
was significantly decreased in PCOS rats compared with the blank control rats (Fig. 6), while DMBG significantly reversed these decreases (Fig. 6). These results demonstrated that PDK-1 and mTOR may be implicated in PCOS, further indicating the involvement of defective insulin signaling and the protective effects of DMBG during the development and treatment of PCOS.

\section{Discussion}

The present study employed letrozole-induced rats that manifested PCOS morphology, androgen excess and obesity, which meets the Rotterdam diagnostic criteria $(1,2)$, and demonstrated that the expression of insulin-mediated PI3K signaling molecules were inhibited in PCOS ovaries, and that DMBG rescued this pathway to a certain degree. These results indicated that the decreased expression of insulin signaling molecules may contribute to the defective PI3K pathway in PCOS ovaries and the protective effects of DMBG on PCOS.

Our previous study detected related PCOS endocrine hormones in serum (23), such as luteinizing hormone, follicle stimulating hormone and estradiol, which indicated hypothalamus-pituitary-gonadal axis dysfunction (23). Therefore, the PCOS model was confirmed by histology and endocrinology in the present study, and the results indicated the presence of numerous follicular cysts in the ovaries and increased testosterone concentrations in the serum of PCOS rats, which was consistent with previous reports (21-23). Hyperandrogenism or androgen excess is the most common endocrine feature of PCOS. Increases in the levels of circulating androgens from the ovary and adrenal gland are not only affected by gonadotropic hormones, but are also affected by insulin and the insulin growth factor system (21-23). Androgen excess may be caused by the degradation of granulosa cells in the follicular cysts of PCOS rats, thus leading to anovulation.

Insulin exerts its effects via the insulin receptor and mediates responses to insulin through phosphorylation of the tyrosine residues of various cellular substrates, particularly IRS proteins. IRS proteins are involved in the regulation of a variety of biological responses as they bind and activate various enzymes and adapter molecules, including IRS-1, IRS-2, IRS-3, IRS-4, GBR2-associated binding protein 1 and p62 (26). IRS-1 and IRS-2 are widely distributed in various mammalian tissues, and commonly included in studies concerning insulin resistance (24-26). However, one study reported that IRS-1 knockout (KO) mice exhibited slower growth and mild resistance to insulin, while disruption of IRS-2 impaired peripheral insulin signaling and pancreatic $\beta$-cell function, and led to the progressive deterioration of glucose homeostasis due to insulin resistance and a lack of $\beta$-cell compensation for insulin resistance (26-28). Furthermore, IRS-2 KO mice were reported to have small, anovulatory ovaries with a reduced numbers of follicles, which led to female infertility (28). In the present study, the protein expression of IRS-2 in the ovaries of PCOS rats was significantly decreased compared with blank control rats, which is consistent with the results of previous studies (27-29). These results further confirm that decreased IRS-2 expression may contribute to insulin resistance in PCOS.

However, changes in the levels of IRS-2 may lead to downstream alterations in insulin signaling (30). The activation of
IRS-2 allows it to bind to the $\mathrm{SH} 2$ domain of the $\mathrm{p} 85$ regulatory subunit of PI3K. The inhibition and activation of PI3K in various tissues has led to differential outcomes $(15,31)$. Decreased PI3K activity was observed in the adipose tissue, which may lead to insulin resistance (11-15). p85 $\alpha$ has an essential role in the activation of PI3K and is an important component in insulin signaling transduction. Furthermore, Wang et al (32) reported that PI3K p85 $\alpha$ was significantly decreased in ovaries from PCOS rats at the mRNA and protein level, and that DMBG enhanced PI3K expression. The results of the present study were consistent with these previous studies. Therefore, the reduced expression of PI3K p $85 \alpha$ may lead to reduced PI3K activity, which subsequently blocks the majority of the metabolic functions of insulin.

PI3K acts as a molecular switch to modulate the activity of a number of serine/threonine-specific kinase cascades that are important in regulating the effects of insulin $(15,31)$. However, upon activation of PI3K, a downstream series of events is induced, which results in the phosphorylation and activation of PDK-1 and mTOR. PDK-1 is a $63 \mathrm{kDa}$ protein kinase that consists of an N-terminal kinase domain and a C-terminal pleckstrin homology domain. It binds to the PI3K products PIP3 and PIP2, which target PDK-1 to the plasma membrane $(33,34)$. mTOR is an evolutionarily conserved serine/threonine kinase that exists as two distinct protein complexes that differ with regards to structure and function, which are termed mTOR complex 1 (mTORC1) and mTORC2. mTOR, as a master integrator, is thought to have a role in ovarian biology. mTOR was reported to regulate the expression of granulosa cell genes in follicles, and mTOR activity may function in follicular growth and ovulation. Furthermore, when was inhibited in preovulatory follicles, human chorionic gonadotropin-induced ovulation was affected (35). To the best of our knowledge, the present study is the first to determine the expression of PDK-1and mTOR in the ovaries of PCOS rats by western blot analysis, and to demonstrate that their expression patterns were similar to those of IRS-2 and PI3K p85a, which indicates that PDK-1 and mTOR expression may be associated with anovulation and insulin resistance in PCOS.

DMBG is normally employed clinically to alleviate PCOS symptoms and to reduce high levels of luteinizing hormone and hyperandrogenism (17-23). However, the underlying mechanism of the effects of DMBG in PCOS remains to be determined. In the present study, the results demonstrated that DMBG partially reversed the expression of IRS-2 and PI3K p85a in PCOS rats. Notably, further analysis of alterations in the protein expression of proteins downstream of PI3K, PDK-1 and mTOR, demonstrated that DMBG partially reversed PCOS-induced decreases in the expression of these two proteins in PCOS ovaries, which may provide a novel research direction to further investigate the molecular mechanisms underlying the regulation of PCOS development and treatment.

In conclusion, the present study demonstrated that defective insulin signaling may occur in PCOS rats, and the protective effects of DMBG on the levels of IRS-2, PI3K, PDK-1 and mTOR in the ovaries of letrozole-induced PCOS rats were reported. Therefore, the results of the present study allows an improved understanding of the potential molecule mechanisms involved in the regulation of the pathophysiology of PCOS, such as the defective insulin-mediated PI3K/mTOR 
signaling, and may provide a theoretical basis for the clinical treatment of PCOS.

\section{Acknowledgements}

The present study was supported by the National Natural Science Foundation of China (grant no. 31271255), the Fujian Provincial Natural Science Foundation (grant nos. 2016J01145 and 2017J01626) and the Fujian Province Science and Technology Project of the Education Department (grant nos. JAT160118 and JZ160426).

\section{References}

1. Broekmans FJ and Fauser BC: Diagnostic criteria for polycystic ovarian syndrome. Endocrine 30: 3-11, 2006.

2. Lane DE: Polycystic ovary syndrome and its differential diagnosis. Obstet Gynecol Surv 61: 125-135, 2006.

3. Makker A, Goel MM, Das V and Agarwal A: PI3K-Akt-mTOR and MAPK signaling pathways in polycystic ovarian syndrome, uterine leiomyomas and endometriosis: An update. Gynecol Endocrinol 28: 175-181, 2012.

4. Zhao Y, Zhang C, Huang Y, Yu Y, Li R, Li M, Liu N, Liu P and Qiao J: Up-regulated expression of WNT5a increases inflammation and oxidative stress via PI3K/AKT/NF- $\kappa \mathrm{B}$ signaling in the granulosa cells of PCOS patients. J Clin Endocrinol Metab 100: 201-211, 2015.

5. Li T, Mo H, Chen W, Li L, Xiao Y, Zhang J, Li X and Lu Y: Role of the PI3K-Akt signaling pathway in the pathogenesis of polycystic ovary syndrome. Reprod Sci 24: 646-655, 2017.

6. Azziz R: Polycystic ovary syndrome, insulin resistance and molecular defects of insulin signaling. J Clin Endocrinol Metab 87: 4085-4087, 2002.

7. Dunaif A: Insulin resistance in women with polycystic ovary syndrome. Fertil Steril 86 (Suppl 1): S13-S14, 2006.

8. Vollenweider P: Insulin resistant states and insulin signaling. Clin Chem Lab Med 41: 1107-1119, 2003.

9. Skrgatić L, Baldani DP, Gersak K, Cerne JZ, Ferk P and Corić M: Genetic polymorphisms of INS, INSR and IRS-1 genes are not associated with polycystic ovary syndrome in Croatian women. Coll Antropol 37: 141-146, 2013.

10. Ciaraldi TP: Molecular defects of insulin action in the polycystic ovary syndrome: Possible tissue specificity. J Pediatr Endocrinol Metab 13 (Suppl 5): S1291-S1293, 2000.

11. Diamanti-Kandarakis E, Argyrakopoulou G, Economou F, Kandaraki E and Koutsilieris M: Defects in insulin signaling pathways in ovarian steroidogenesis and other tissues in polycystic ovary syndrome (PCOS). J Steroid Biochem Mol Biol 109: 242-246, 2008

12. Book CB and Dunaif A: Selective insulin resistance in the polycystic ovary syndrome. J Clin Endocrinol Metab 84: 3110-3116, 1999.

13. Wu XK, Zhou SY, Liu JX, Pöllänen P, Sallinen K, Mäkinen M and Erkkola R: Selective ovary resistance to insulin signaling in women with polycystic ovary syndrome. Fertil Steril 80: 954-965, 2003.

14. Venkatesan AM, Dunaif A and Corbould A: Insulin resistance in polycystic ovary syndrome: Progress and paradoxes. Recent Prog Horm Res 56: 295-308, 2001.

15. Shah KN and Patel SS: Phosphatidylinositide-3 kinase: A newer molecular target in metabolic and hormonal pathway of polycystic ovary syndrome. Exp Clin Endocrinol Diabetes 122: 261-267, 2014.

16. Saltiel AR and Pessin JE: Insulin signaling pathways in time and space. Trends Cell Biol 12: 65-71, 2002.
17. Tan MH, Alquraini H, Mizokami-Stout $\mathrm{K}$ and MacEachern $\mathrm{M}$ : Metformin: From research to clinical practice. Endocrinol Metab Clin North Am 45: 819-843, 2016.

18. Genazzani AD, Ricchieri F and Lanzoni C: Use of metformin in the treatment of polycystic ovary syndrome. Womens Health (Lond) 6: 577-593, 2010.

19. FruzzettiF,Perini D, Russo M,BucciFand Gadducci A: Comparison of two insulin sensitizers, metformin and myo-inositol, in women with polycystic ovary syndrome (PCOS). Gynecol Endocrinol 33: 39-42, 2017

20. Tan X, Li S, Chang Y, Fang C, Liu H, Zhang X and Wang Y: Effect of metformin treatment during pregnancy on women with PCOS: A systematic review and meta-analysis. Clin Invest Med 39: E120-E131, 2016.

21. Genazzani AD, Strucchi C, Luisi M, Casarosa E, Lanzoni C, Baraldi E, Ricchieri F, Mehmeti H and Genazzani AR: Metformin administration modulates neurosteroids secretion in non-obese amenorrhoic patients with polycystic ovary syndrome. Gynecol Endocrinol 22: 36-43, 2006.

22. Genazzani AD, Lanzoni C, Ricchieri F, Baraldi E, Casarosa E and Jasonni VM: Metformin administration is more effective when non-obese patients with polycystic ovary syndrome show both hyperandrogenism and hyperinsulinemia. Gynecol Endocrinol 23: 146-152, 2007.

23. Wang F, Zhang Z, Wang Z, Xiao K, Wang Q, Su J and Wang Z: Expression and clinical significance of the HIF-1a/ET-2 signaling pathway during the development and treatment of polycystic ovary syndrome. J Mol Histol 46: 173-181, 2015.

24. Palomba S, Falbo A, Zullo F and Orio F Jr: Evidence-based and potential benefits of metformin in the polycystic ovary syndrome: A comprehensive review. Endocr Rev 30: 1-50, 2009.

25. Li X, Guo YR, Lin JF, Feng Y, Billig H and Shao RJ: Combination of diane-35 and metformin to treat early endometrial carcinoma in PCOS women with insulin resistance. J Cancer 5: 173-181, 2014.

26. White MF: The IRS-signaling system: A network of docking proteins that mediate insulin and cytokine action. Recent Prog Horm Res 53: 119-138, 1998.

27. Withers DJ, Gutierrez JS, Towery H, Burks DJ, Ren JM, Previs S, Zhang Y, Bernal D, Pons S, Shulman GI, et al: Disruption of IRS-2 causes type 2 diabetes in mice. Nature 391: 900-904, 1998.

28. Kubota N, Tobe K, Terauchi Y, Eto K, Yamauchi T, Suzuki R, Tsubamoto Y, Komeda K, Nakano R, Miki H, et al: Disruption of insulin receptor substrate 2 causes type 2 diabetes because of liver insulin resistance and lack of compensatory beta-cell hyperplasia. Diabetes 49: 1880-1889, 2000.

29. Burks DJ, Font de Mora J, Schubert M, Withers DJ, Myers MG, Towery HH, Altamuro SL, Flint CL and White MF: IRS-2 pathways integrate female reproduction and energy homeostasis. Nature 407: 377-382, 2000.

30. Mlinar B, Marc J, Janez A and Pfeifer M: Molecular mechanisms of insulin resistance and associated diseases. Clin Chim Acta 375: 20-35, 2007.

31. Shepherd PR, Withers DJ and Siddle K: Phosphoinositide 3-kinase: The key switch mechanism in insulin signalling. Biochem J 333: 471-490, 1998.

32. Wang LH, Wang X, Yu XZ and Xu WT: Potent therapeutic effects of shouwu jiangqi decoction on polycystic ovary syndrome with insulin resistance in rats. Chin J Integr Med 22: 116-123, 2016.

33. Gagliardi PA, di Blasio L and Primo L: PDK1: A signaling hub for cell migration and tumor invasion. Biochim Biophys Acta 1856: 178-188, 2015.

34. Li Y, Yang KJ and Park J: Multiple implications of 3-phosphoinositide-dependent protein kinase 1 in human cancer. World J Biol Chem 1: 239-247, 2010.

35. Siddappa D, Kalaiselvanraja A, Bordignon V, Dupuis L, Gasperin BG, Roux PP and Duggavathi R: Mechanistic target of rapamycin (MTOR) signaling during ovulation in mice. Mol Reprod Dev 81: 655-665, 2014. 